\title{
Sub-femtomolar detection of DNA and discrimination of mutant strands using microwell-array assisted digital enzyme-linked oligonucleotide assay
}

\author{
Lisa Tripodi ${ }^{1}$, Daan Witters ${ }^{1}$, Tadej Kokalj ${ }^{1,2}$, Heinrich J. Huber ${ }^{3}$, Robert Puers ${ }^{4}$, Jeroen Lammertyn ${ }^{1 *}$, \\ Dragana Spasic ${ }^{1}$ \\ ${ }^{1}$ BIOSYST-MeBioS, KU Leuven, Leuven, Belgium, \\ ${ }^{2}$ Institute of Metals and Technology, Ljubljana, Slovenia, ${ }^{3}$ Institute for Automation Engineering, University of \\ Magdeburg, Germany, ${ }^{4}$ ESAT-MICAS, KU Leuven, Belgium \\ *jeroen.lammertyn@biw.kuleuven.be
}

\begin{abstract}
Detection methods that do not rely on the amplification of DNA but can reach sensitivity, specificity and throughput of gold standard methods, such as $\mathrm{qPCR}$, have been extensively explored in recent years. Here, we present a hydrophilic-in-hydrophobic $(\mathrm{HIH})$-microwell array platform that empowers a panel of different amplification-free DNA bioassays: digital enzyme-linked oligonucleotide assay (ELONA), ligation-assisted (LA) digital ELONA and so-called 'analog' bioassays. We developed all three bioassays by using magnetic beads for capturing DNA target, followed by hybridization of enzymelabelled detection probes and sealing of the built complexes into the femtoliter HIH microwells to achieve the fluorescent readout of single DNA molecules. With the optimized digital ELONA bioassay, we successfully detected 97 and 200 nt-long ssDNA molecules down to 68 and 92 aM, respectively, demonstrating extremely high sensitivity of the bioassay and its flexibility towards targets of different lengths. Importantly, we also proved that the same bioassay concept was suited to detect substantially higher concentrations of ssDNA (up to picomolar levels) by quantifying the total fluorescent intensity rather than counting fluorescent events for digital quantification. Finally, we
\end{abstract}


advanced this concept towards LA digital ELONA capable of differentiating wildtype strands from those carrying single-point mutations even when the former were constituting only $1 \%$ of the DNA mixture and were present at $2 \mathrm{fM}$ concentration. In conclusion, the developed platform showed remarkably high sensitivity, specificity and versatility for amplification-free detection of DNA and as such can be valuable for numerous applications in medical diagnostics, gene analysis, food safety and environmental monitoring.

Keywords: Digital enzyme-linked oligonucleotide assay (ELONA), DNA, single nucleotide polymorphisms, sensitivity, specificity, hydrophilic-in-hydrophobic (HIH) microwell array

\section{Introduction}

Detection of DNA and single nucleotide polymorphisms (SNPs) is of paramount importance in medical diagnostics (e.g. diagnosis of viral and bacterial infections [1-3], cancer [4] and chronic lymphocytic leukemia [5]), gene analysis (detection of SNPs for diagnosing sickle cell disease and cystic fibrosis [67]), food safety (e.g. pathogen and allergen detection $[8,9])$ and environmental monitoring (e.g. evolutionary responses to pollution and global warming [10] and biodiversity [11]). Although numerous detection approaches exist already, they all strongly differ in terms of performance (sensitivity, specificity, throughput) and cost, the latter being a major limiting factor for conventional and whole exome sequencing (WES), despite their high detection sensitivity and capacity to identify genetic variants as SNPs $[12,13]$. Furthermore, the majority of DNA and SNPs detection techniques are still based on DNA amplification, including the golden standard in the field, such as real-time quantitative polymerase chain reaction (qPCR) [14] or some newer methods, like ligase chain reaction 
(LCR) [15] and rolling circle amplification (RCA) [16-18]. Despite their unquestionable importance, amplification-based detection techniques face several limitations due to the fact that used enzymes are: (i) susceptible to inhibition by sample components, which often leads to false negative results [19], (ii) expensive and highly susceptible to assay conditions (i.e. temperature, surfactants) and (iii) often error-prone, leading to amplification of non-target sequences and false positive results [20].

As a consequence, other methods have been investigated, which do not rely on the replication of DNA but rather on direct detection of the target molecules. Recently, Song et al. have demonstrated the use of SiMoA ${ }^{\mathrm{TM}}$ technology for the direct detection of low concentrations of DNA (as low as 70 attomolar) by hybridization between the target strand and complementary probes [21]. The general approach of $\mathrm{SiMoA}^{\mathrm{TM}}$ technology relies on capturing target molecules (either protein or oligonucleotides) on a superparamagnetic bead and their subsequent labeling with an enzyme to achieve detection, similar to a classical enzyme-linked immunosorbent assay (ELISA) or enzyme-linked oligonucleotide assay (ELONA) [22-24]. In a next step, the individual beads are isolated in microwells in the presence of the proper enzymatic substrate for the enzyme and sealed by oil. The microwell arrays are then imaged at the emission wavelength of the fluorescent enzymatic product to determine the fraction of beads with a fluorescent signal. According to Poisson statistics, at low concentrations, where only a fraction of the microwells exhibits the fluorescent signal, the majority of fluorescent beads are associated with only single target molecule [25]. In this case, the target molecules in the sample can be literally counted, which is known as a digital bioassay concept [26-28].

Despite significant sensitivity of digital ELONA achieved with the SiMoA ${ }^{\mathrm{TM}}$, loading efficiency of magnetic beads on this [29] or other reported microwell-based platforms [30] is only $40-60 \%$. To further improve this loading efficiency, our group recently introduced hydrophilic-in-hydrophobic (HIH) Teflon microwells on the digital microfluidics (DMF) platform that could support bead-based digital bioassay implementation [31]. The use of HIH microwells increased the loading efficiency of 
beads to almost $100 \%$ in addition to speeding up the transfer of individual beads into the microwells and facilitating the sealing of reagents in the wells (due to wettability difference between the hydrophilic well bottom and hydrophobic surface area surrounding the wells). By using this $\mathrm{HIH}-$ microwell arrays, our group already reported the detection of attomolar concentrations (10 aM) of beta-galactosidase enzymes on DMF chips [31] and more recently femtomolar concentrations (4 fM) of Influenza A Nucleoprotein [32] as well as attomolar concentrations of Alzheimer's disease biomarker Tau (24 aM in buffer and 55 aM in blood plasma) [33].

To further explore the great potential of these HIH-microwell arrays, in the present work, we implement for the first time the bead-based digital ELONA concept for detecting not only single stranded (ss) DNA but SNPs as well. In this assay, target ssDNA is first captured on superparamagnetic beads through hybridization with complementary capture probes immobilized on the beads surface, followed by labelling with enzyme-linked detection probes for fluorescent detection. Using this assay, we establish calibration curves for ssDNA molecules of two different lengths, being 97 and 200 nucleotides (nt). Moreover, we examine the capacity of the HIH-microwell array platform to quantify a wide range of ssDNA concentrations, spanning from $0.1 \mathrm{fM}$ up to $10 \mathrm{pM}$. Finally, we use for the first time digital ELONA technology to discriminate wildtype (WT) DNA strands from those carrying SNPs (mutant, MT), thereby integrating the needs for sensitive, specific and high throughput detection of both DNA molecules and SNPs on a single platform. 


\section{Materials and methods}

\subsection{Materials and reagents}

The following reagents have been purchased from Sigma-Aldrich (Bornem, Belgium): fluorescein di( $\beta$ D-galactopyranoside) (FDG), bovine serum albumin (BSA), $\mathrm{MgCl}_{2}, 2$-(N-Morpholino) ethanesulfonic acid hydrate (MES), phosphate buffered saline (PBS), Tween 20, 2-Amino-2-(hydroxymethyl)-1,3propanediol (Trizma ${ }^{\circledR}$ base), $\mathrm{NaCl}$, trisodium citrate (dehydrate), dextran sulfate and Pluronic F68. Superparamagnetic beads (Lodestar, $2.7 \mu \mathrm{m}$ diameter) with carboxylic acid groups were purchased from Agilent (Diegem, Belgium). Superblock blocking buffer and 1-ethyl-3-(3-dimethylaminopropyl) carbodiimide (EDC) were purchased from Thermo Scientific (Waltham, MA, USA). All DNA strands were synthesized by Integrated DNA Technologies (IDT, Haasrode, Belgium) and the overview of sequences is given in Table 1.

\begin{tabular}{|c|c|c|c|}
\hline Probe Name & Sequence (5'- 3') & Length & Modification \\
\hline \multicolumn{4}{|l|}{ TARGETS } \\
\hline Target_WT (97) & $\begin{array}{l}\text { AGG CCT TTA CCC CAC CAA CTA GCT AAT CCG ACC TAG GCT } \\
\text { CAT CTG ATA GCG TGA GGT CCG AAG ATC CCC CAC TTT CTC } \\
\text { CCT CAG GAC GTA TGC GGT A }\end{array}$ & $97 \mathrm{nt}$ & none \\
\hline Target_WT (200) & $\begin{array}{l}\text { GCG CGC GGA TCC CCT GGA ACC TCA CAT CAC ATC AAC GCG } \\
\text { CAG ATC ATG CAG CTG CAC CAC AGC AAG CAC CAC GCG GCC } \\
\text { TAC GTG AAC AAC CTG AAC GTC ACC GAG GAG AAG TAC CAG } \\
\text { GAG GCG TTG GCC AAG GGA GAT GTT ACA GCC CAG ATA GCT } \\
\text { CTT CAG CCT GCA CTG AAG TTC AAT GGT GGT GGT CAT AAT } \\
\text { CAA TCA TAG CA }\end{array}$ & $200 \mathrm{nt}$ & none \\
\hline Target_MT (97) & $\begin{array}{l}\text { AGG CCT TTA CCC CAC CAA CTG GCT AAT CCG ACC TAG GCT } \\
\text { CAT CTG ATA GCG TGA GGT CCG AAG ATC CCC CAC TTT CTC } \\
\text { CCT CAG GAC GTA TGC GGT A }\end{array}$ & $97 \mathrm{nt}$ & none \\
\hline \multicolumn{4}{|l|}{ CAPTURE PROBES } \\
\hline Capture probe_WT (97) & AGT TGG TGG GGT AAA GGC CTC TCT CTC TCT & $30 \mathrm{nt}$ & 3' amino \\
\hline Capture probe_WT (200) & $\begin{array}{l}\text { TCT GCG CGT TGA TGT GTG GTT CCA GGG GAT CCG CGC GCC } \\
\text { TCT CTC TCT }\end{array}$ & $47 \mathrm{nt}$ & 3' amino \\
\hline Capture probe_LIG (97) & AGT TGG TGG GGT AAA GGC CTC TCT CTC TCT & $30 \mathrm{nt}$ & $\begin{array}{l}\text { 3' amino } \\
\text { 5' phosphate }\end{array}$ \\
\hline \multicolumn{4}{|l|}{ DETECTION PROBES } \\
\hline Detection probe 1_WT (97) & TTT TCT AGG TCG GAT TAG C $\underline{\mathbf{T}}$ & $20 \mathrm{nt}$ & 5' biotin-TEG \\
\hline Detection probe 2_WT (97) & GAT CTT CGG ACC TCA & $15 \mathrm{nt}$ & 5' biotin-TEG \\
\hline Detection probe 3_WT (97) & GCA TAC GTC CTG AGG & $15 \mathrm{nt}$ & 5' biotin-TEG \\
\hline Detection probe 1_WT (200) & GTT CAG GTT GTT CAC GTA GGC CGC GTG GTG CTT GCT GT & $38 \mathrm{nt}$ & 5' biotin-TEG \\
\hline Detection probe 2_WT (200) & GCT ATC TGG GCT GTA ACA TCT CCC TTG GCC AAC GC & $35 \mathrm{nt}$ & 5' biotin-TEG \\
\hline Detection probe 3_WT (200) & GCG CCT CGA GTG CTA TGA TTG ATA TGA CCA CCA CC & $35 \mathrm{nt}$ & 5' biotin-TEG \\
\hline
\end{tabular}


Table 1: Overview of DNA sequences used in the paper: targets, capture and detection probes. WT is an abbreviation for the wildtype sequences (97 and $200 \mathrm{nt}$ long, respectively), while MT (97) is an abbreviation for the mutant sequence differing from the WT (97) in one SNP (here indicated with in bold). The bold and underlined nucleotides represent the site of ligation. Sequence of Target_WT (97) is specific for the bacterium Pseudomonas aeruginosa, while sequence of Target_WT (200) is of human origin. Both sequences are selected here purely as a model systems for development of digital ELONA. Capture probe_LIG (97) is modified with a phosphate group at its 5' end to allow ligation with the detection probe in the ligation assays (Section 2.6).

Streptavidin-conjugated $\beta$-galactosidase (S $\beta$ G) enzyme was acquired from Life Technologies (Carlsbad, CA, USA). Photolithography reagents were obtained from Rohm and Haas (Marlborough, MN, USA) and Parylene-C dimer was purchased from Plasma Parylene Coating Services (Rosenheim, Germany). Teflon-AF $^{\circledR}$ (6 \% w/w in FC-40) was obtained from Dupont (Wilmington, DE, USA). Dynasylan F 8263 was a kind gift from Evonik Degussa International AG (Essen, Germany). Vapor-Lock oil was obtained from Qiagen (Hilden, Germany). Ampligase Thermostable DNA Ligase $(5 \mathrm{U} / \mu \mathrm{L})$ and the corresponding buffer were purchased from Westburg (Leusden, The Nederlands).

\subsection{Fabrication of HIH-microwell arrays}

The HIH-microwell arrays consist of a glass slide covered with a layer of Teflon, which was patterned to accommodate one individual bead per microwell as previously described [31]. The micro-pattern is composed of 62.500 femtoliter-sized microwells of $4.5 \mu \mathrm{m}$ diameter, $3 \mu \mathrm{m}$ depth and center to center distance of $8 \mu \mathrm{m}$ in a square patch of $2 \times 2 \mathrm{~mm}^{2}$. Briefly, the microfabrication of the wells was done as follows: first, a squared glass slide $(50 \mathrm{~mm} \times 50 \mathrm{~mm} \times 1 \mathrm{~mm}$ ) was spin-coated with a $3 \mu \mathrm{m}$ thick Teflon layer after prior deposition of Dynasylan F 8263. On top of that, a thin Parylene-C layer was deposited through chemical vapor deposition and used to protect the Teflon surface during the patterning 
process. Next, an aluminum hard mask of $100 \mathrm{~nm}$ was deposited through thermal evaporation and patterned according to classical photolithographic and wet chemical etching steps. Once patterning in the aluminum mask was completed, the underlying Parylene- $\mathrm{C}$ and Teflon were etched with oxygen plasma until the glass. Finally, the Parylene-C protective mask was peeled-off together with the aluminum mask thus leaving behind a hydrophobic pattern in Teflon with a hydrophilic-glass bottom (i.e. HIH microwells).

\subsection{Functionalization of magnetic beads with DNA capture probes}

Superparamagnetic carboxyl beads (Lodestar, $2.7 \mu \mathrm{m}$ diameter) were functionalized with capture probes according to manufacturer's instructions. Capture probes of 30 and $47 \mathrm{nt}$ were designed to hybridize to specific parts of the 97 and 200 nt long ssDNA targets, respectively (see Table 1). In addition, the capture probes were modified with an amino group (-NH2) at the $3^{\prime}$ end for facilitating binding to the $\mathrm{COOH}$-group of beads through EDC surface chemistry.

Prior to functionalization with DNA capture probes, $100 \mu \mathrm{L}$ beads $(30 \mathrm{mg} / \mathrm{mL}$ concentration, which corresponds to $2.3 \times 10^{5}$ beads) was washed twice in $100 \mu \mathrm{L}$ MES buffer ( $25 \mathrm{mM}, \mathrm{pH}$ 5) for $10 \mathrm{~min}$ on a rotating platform at room temperature (RT). After washing, the supernatant was removed and 2.1 $\mathrm{nmol}$ of capture probes was added to the beads in $28 \mu \mathrm{L}$ MES buffer ( $25 \mathrm{mM}, \mathrm{pH}$ 5). This DNA-bead solution was incubated in the rotating platform for $30 \mathrm{~min}$ under slow rotation. Just before use, EDC was dissolved in MES buffer (100 mM, pH 5) at a $100 \mathrm{mg} / \mathrm{mL}$ concentration and added to the beads/DNA suspension to a final volume of $100 \mu \mathrm{L}$. After mixing, the solution was incubated overnight with slow rotation. Subsequently, non-reacted carboxylic acid groups of the beads were quenched by incubating the beads in Tris-HCl buffer (100 mM, pH 7.4) for 1 hour at RT. Next, the coated beads were incubated with $100 \mu \mathrm{L}$ of commercial Superblock blocking buffer to reduce non-specific binding of biomolecules during the experiment. Finally, beads functionalized with DNA capture probes were 
resuspended in $200 \mu \mathrm{L}$ of Superblock buffer with $0.05 \%$ Tween 20 and used for digital ELONA as described in section 2.4 .

\subsection{Single DNA molecule detection with digital ELONA}

For single DNA molecule detection with bead-based digital ELONA, three detection probes were designed to hybridize with specific parts of the ssDNA targets (i.e. 97 and 200-nt long DNA targets, Figure $1 \mathrm{~A})$. Each detection probe was functionalized with a biotin-TEG group on the $5^{\prime}$ site to enable binding of $S \beta G$ enzyme for signal generation. Sequences of both targets and their respective detection probes are listed in Table 1.

A)

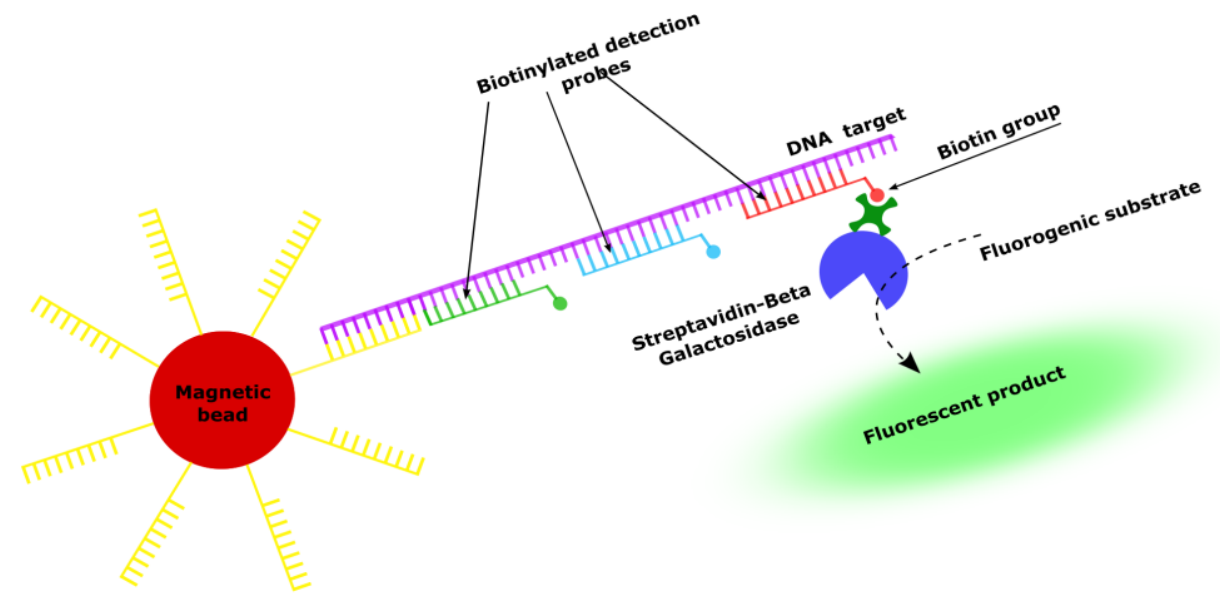

B)

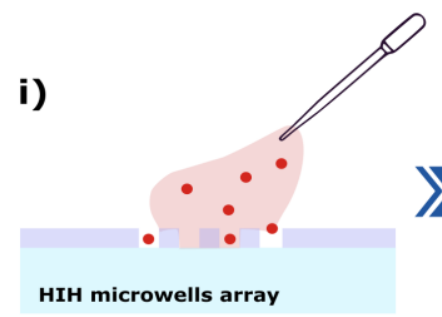

ii)

Oil

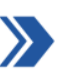

HIH microwells array iii)

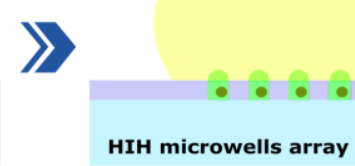

\section{Magnet}

iv)

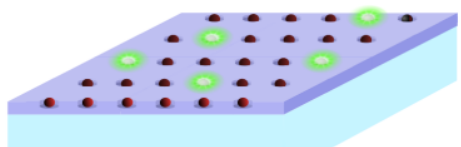

HIH microwells array v)

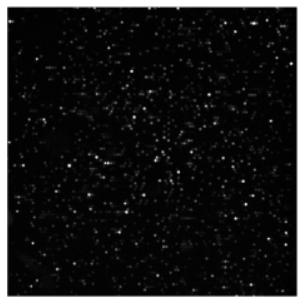


Figure 1: Schematic overview of the digital ELONA concept on the HIH-microwell array platform. A) DNA hybridization complex scheme: a functionalized magnetic particle captures a single DNA molecule, which is hybridized to biotinylated complementary detection probes and labelled with a SBG-enzyme. B) Schematic overview of sample manipulation on the HIH-microwell array: i) The droplet containing the beads is shuttled over the array with a pipet tip allowing beads seeding by using a magnet. The sample droplet is then removed thus leaving each bead inside a single microwell. ii) FDG substrate is sealed on top of the wells and coated with an oil shell. iii) Excess of FDG substrate is removed while the oil shell embeds femtoliter FDG droplets in the microwells. iv) Schematic overview of the entire HIH-microwell array with several fluorescent events that allow for the digital counting of single DNA molecules. v) Example of a binary image of the fluorescent spots obtained with the Image J Software.

To perform digital ELONA, ssDNA target was diluted to the desired concentration $(100,10,1,0.5$ and $0.1 \mathrm{fM}$ ) in $5 x$ SSC buffer (saline-sodium citrate buffer) with $10 \%$ dextran sulfate and $0.1 \%$ Tween 20. Subsequently, $350 \mu \mathrm{L}$ of target DNA was mixed with $50 \mu \mathrm{L}$ of each of the three detection probes in a final volume of $500 \mu \mathrm{L}$, resulting in a $0.5 \mathrm{nM}$ concentration for each detection probe. This mixture was vortexed, heated up to $95^{\circ} \mathrm{C}$ for $7 \mathrm{~min}$ and quickly cooled on ice. Next, $1.8 \mu \mathrm{L}$ of superparamagnetic beads $\left(2.5 \times 10^{5}\right)$, functionalized with capture probes (see section 2.3 ), were added to the sample, mixed, and incubated for $90 \mathrm{~min}$ at $42{ }^{\circ} \mathrm{C}$ in order to form the DNA complexes. After incubation, beads were washed 3 times with $0.2 x$ SSC buffer, and subsequently incubated with 200 pM solution of S $\beta$ G in Superblock buffer containing $0.05 \%$ Tween 20 for 30 min at $23{ }^{\circ} \mathrm{C}$. Next, beads were washed thoroughly 8 times in 5x PBS buffer with $0.1 \%$ Tween 20. Finally, beads were resuspended in $10 \mu \mathrm{L}$ of PBS buffer containing $0.05 \%$ Pluronic F68 and seeded on HIH-microwell array as described in the following section. 


\subsection{Seeding of beads on $\mathrm{HIH}$-microwell array and image processing}

Magnetic beads with built DNA complexes were seeded on HIH-microwell arrays following a similar procedure described by Leirs et al. (Figure 1B) [32]. A $5 \mu \mathrm{L}$ sample droplet containing the beads was manually shuttled back and forth over the HIH-microwell array using a pipette tip. During the seeding process, the $\mathrm{HIH}$-microwell array was placed on top of a magnet ( $\mathrm{NdFeB}, 16 \mathrm{~mm}^{2}$ area, $12.7 \mathrm{~N}$, Supermagnete, Germany) at a distance of $1 \mathrm{~mm}$. After the beads were seeded in the microwells, the sample droplet was removed and a $30 \mu \mathrm{L}$ droplet $(152 \mu \mathrm{M})$ of FDG substrate was brought on top of the array. The microwells were sealed by applying a $150 \mu \mathrm{L}$ droplet of Vapor-Lock oil on top of the FDG droplet and carefully pipetting away the droplet of FDG substrate from the array, thus creating femtoliter-sized reaction wells. The fluorescent product generated by SBG enzyme was visualized using a fluorescence microscope (IX71 Olympus Corporation, Japan) equipped with a mercury lamp, 20x objective and EM-CCD camera (Hamamatsu Photonics K.K., Japan). The fluorescence was recorded from the bottom of the transparent $\mathrm{HIH}$-microwell array before the start of any possible enzymatic reaction inside the microwells (at $0 \mathrm{~min}$ ) to correct for the autofluorescence of the beads and to subtract from another image taken after $20 \mathrm{~min}$. The following emission/excitation wavelength, dichromatic and density filters were used for imaging: 1) WIBA filter (excitation 460-495 nm, emission 510-550 nm), 2) dichromatic filter (DM505) and 3) Neutral density filters (3\%). Obtained binary images were used to count all the fluorescent spots with the Image J software (US National Institutes of Health, http://rsb.info.nih.gov/ij/) by cutting off image noise with appropriate threshold and through the command "find maxima". After the enzymatic reaction, a last image was taken in order to visualize and count all the seeded beads. In this case the following filters were used: 1) WIGA filter (excitation 530-550 nm, emission 575-625 nm), and dichromatic filter DM570 in combination 
with a light intensity of $100 \%$. The results are presented as the percentage of active beads, which is calculated by normalizing the number of fluorescent wells to the total number of seeded beads.

\subsection{SNP detection in DNA mixtures with digital ELONA}

For SNP detection with digital ELONA, Target_WT (97) and Target_MT (97) (sequences shown in Table 1) were first separately diluted in a 5x SSC buffer containing $10 \%$ dextran sulfate and $0.1 \%$ Tween 20. Subsequently, DNA mixtures were prepared by mixing WT and MT DNA to reach 2, 20 and $100 \mathrm{fM}$ final concentration of WT sequences in a sample with total DNA concentration of $200 \mathrm{fM}$. Then, $350 \mu \mathrm{L}$ of each DNA mixture was incubated with $100 \mu \mathrm{L}$ of a DNA solution containing single detection probe (5 nM, Detection probe 1_WT(97)). Moreover, two controls were prepared: control 1 (350 $\mu \mathrm{L}$ of 5x SSC buffer containing $10 \%$ dextran sulfate and $0.1 \%$ Tween 20 with $100 \mu \mathrm{L}$ DNA detection probe $(5 \mathrm{nM}))$ and control 2 (350 $\mu \mathrm{L}$ of a pure MT DNA solution (100 fM) incubated with $100 \mu \mathrm{L}$ of DNA detection probe $(5 \mathrm{nM})$ ). All the samples were heated at $95{ }^{\circ} \mathrm{C}$ for $7 \mathrm{~min}$ before being cooled on ice for $5 \mathrm{~min}$. Then, $1.8 \mu \mathrm{L}$ of magnetic beads $\left(2.5 \times 10^{5}\right)$ functionalized with capture probes (as described in section 2.3) was added to the sample and incubated at $42{ }^{\circ} \mathrm{C}$ on a thermomixer at $750 \mathrm{rpm}$ for $90 \mathrm{~min}$. Next, the DNA complex captured on beads was washed three times in $100 \mu \mathrm{L}$ of $0.2 x$ SSC buffer. Immediately after removing the washing buffer, a $20 \mu \mathrm{L}$ solution containing $10 \mathrm{U}$ Ampligase enzyme, Ampligase buffer (Ampligase, Thermostable DNA ligase, Epicentre) and $4 \mu \mathrm{g}$ of BSA were added to each sample.

In order to achieve a proper ligation between the capture and the detection probes when capturing the WT sequence, the solutions were incubated for $5 \mathrm{~min}$ at $50^{\circ} \mathrm{C}$ in a thermomixer at 750 rpm as described in the Supplementary information (Figure S4). Then, the DNA mixtures were heated up to $65{ }^{\circ} \mathrm{C}$ and washed in 5x PBS with $0.05 \%$ Tween 20, which was repeated three times. Following the buffer removal, the DNA-beads complex was incubated with a $200 \mathrm{pM}$ solution of S $\beta$ G for 30 min. 
Finally, the DNA-beads complexes were washed eight times with 5x PBS buffer containing $0.1 \%$ Tween 20 and resuspended in a $10 \mu \mathrm{L}$ of Superblock buffer right before being seeded and characterized on the HIH-microwell arrays.

\section{Results and discussion}

\subsection{Establishing digital ELONA on HIH-microwell array platform}

To detect single DNA molecules without amplification, we used our previously published HIH-microwell array platform [31-33] and established a bead-based digital ELONA. As already described in sections 2.4 and 2.5 (and schematically depicted in Figure 1), in the digital ELONA concept, superparamagnetic beads were first functionalized with capture probes, specifically designed to capture target ssDNA through hybridization. Biotinylated detection probes were then hybridized to the target strand and labelled with an SBG enzyme. Subsequently, the beads with built DNA complexes were seeded with single bead resolution on a $\mathrm{HIH}$-microwell array platform. This was followed by printing and sealing femtoliter FDG solution over every bead in an oil matrix. FDG substrate was finally converted by S $\beta$ G enzyme into fluorescent product only in the presence of a captured DNA target to result in a fluorescent signal that was microscopically detected.

We first established the digital ELONA concept by using 97-nt long ssDNA target (referred to as Target_WT (97)). The sequence of Target_WT (97) originates from the bacterium Pseudomonas aeruginosa and is used in this paper purely as a model system. Initially, the target ssDNA was heated to $95^{\circ} \mathrm{C}$ in the presence of the different DNA detection probes and quickly cooled on ice. This was done in order to minimize the presence of any possible secondary structures due to DNA self-folding and thereby to maximize capturing efficiency of target DNA molecules on the magnetic beads.

Because the Target_WT (97) is $97 \mathrm{nt}$ long, it offers the possibility to hybridize with multiple short detection probes (up to $20 \mathrm{nt}$ long). Therefore, we wanted to first evaluate the correlation between 
the sensitivity of the digital ELONA and the number of detection probes used in the assay. The use of three detection probes increased the sensitivity of the assay substantially compared to the use of one detection probe (Figure 2A). There are two possible explanations for this observation: (i) an increase in the total number of $S \beta G$ enzymes bound to the DNA complex, or (ii) an increased probability of binding a single SBG enzyme due to the greater availability of biotin binding sites on a single DNA complex. Although the first option cannot be completely excluded, this was unlikely because the distance between the different biotin groups (coupled to the detection probes) is probably too small $(\sim 4 \mathrm{~nm})$ for binding more than one $S \beta G$ enzyme $(\sim 11 \mathrm{~nm}, 520 \mathrm{kDa})$ per target DNA strand. Therefore, the increased sensitivity is more likely caused by the higher number of biotin groups when using more than one detection probe and the increased probability of successfully binding an SBG enzyme. This is in agreement with previously reported work from Song et al., suggesting that the increase in number of detection probes should improve the binding chances of the $S \beta G$ enzyme to the biotinylated strands and, consequently, the detection sensitivity [21]. Taking these results into account, all digital ELONA experiments were performed with three detection probes. Importantly, in order to achieve labelling of every captured molecule, the selected concentration of detection probes $(5 \mathrm{nM})$ was much higher than the femto- and sub-femtomolar concentrations of target DNA we aimed to detect.

Next, we evaluated the concentration of S $\beta G$ enzyme for obtaining the highest signal-to-noise ratio as well as the effect of adding dextran sulfate on the hybridization efficiency. As shown in Figure S1 (Supplementary information), 200 pM of S $\beta$ G enzyme resulted in the highest signal-to-noise ratio for detecting Target_WT (97) and has been used in all experiments. Moreover, adding dextran sulfate to the hybridization buffer resulted in higher sensitivity, especially for femtomolar $(0-100 \mathrm{fM})$ concentrations of target ssDNA (Figure S2, Supplementary information) next to enabling a short hybridization time (90 min). Therefore, dextran sulfate was added to the hybridization buffer for all experiments. 
Taking into account all the above-mentioned optimized parameters, different concentrations of the Target_WT (97) were measured in the femtomolar range $(0-100 \mathrm{fM})$ using digital ELONA. The obtained log-log plot for the entire range is presented in Figure $2 \mathrm{~B}$, whereas a linear calibration curve in the sub-femtomolar range ( 0 to $1 \mathrm{fM}$ ) is depicted in Figure $2 \mathrm{C}$. The limit of detectable concentration $\left(L O D_{x}\right)$ was calculated by fitting Equation 1 (Eq.1):

$$
L O D_{y}=\mathrm{a}+\mathrm{b} * L O D_{x} \quad(E q .1)
$$

and putting $L O D_{y}$ (limit of detectable signal) equal to the sum of the mean background signal value (i.e. blank) and 3 times the standard deviation of the blank based on three independent repetitions. The $\mathrm{LOD}_{x}$ was calculated to be $0.068 \mathrm{fM}(68 \mathrm{aM})$.

A)

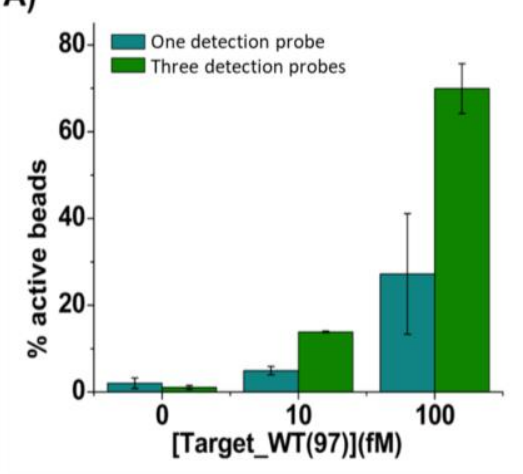

B)

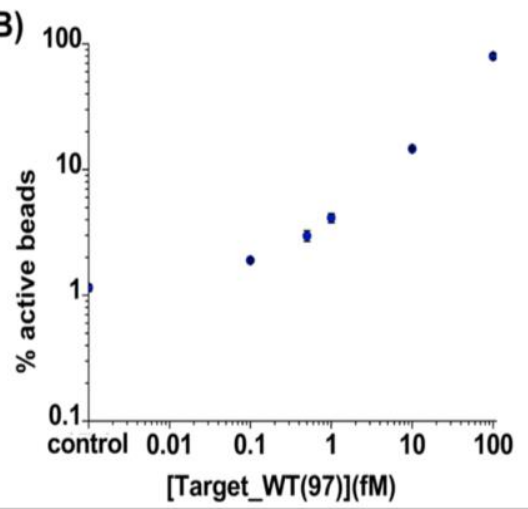

C)

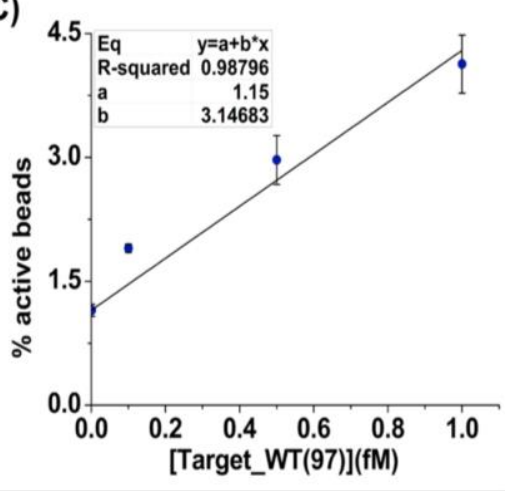

Figure 2: A) Percentage of active beads shown for different Target_WT (97) concentrations (0, 10 and $100 \mathrm{fM})$ in the presence of one (Detection probe 1_WT (97)) and three detection probes. B) Log-log plot of the percentage of active beads in function of the femtomolar concentrations of the target. C) Calibration curve representing the percentage of active beads in function of sub-femtomolar target concentrations. The control signal (1.15\%) corresponds to the non-specific binding of S $\beta G$ enzyme to the magnetic beads at $0 \mathrm{fM}$ target concentration. Error bars are standard deviations based on three independent repetitions. 


\subsection{Expanding digital ELONA towards single molecule detection of longer DNA molecules}

Next, we wanted to test how robust the established digital ELONA is towards detecting longer DNA molecules. To do this, we used 200-nt long DNA strands of human origin as a model system (referred to as Target_WT (200)) and designed target specific capture and detection probes. Both types of probes were longer than those used for Target_WT (97), with lengths ranging from 35 to $47 \mathrm{nt}$ (Detection probes 1,2,3_WT (200), Table 1), in order to limit the formation of the target secondary structures and thereby increase strand stiffness and stability. Similar to the Target_WT (97), using three detection probes resulted in higher sensitivity compared to the assay with only one detection probe (Figure 3A). Different concentrations (0 - $100 \mathrm{fM}$ ) of Target_WT (200) were measured using digital ELONA and the obtained loglog plot for the entire range is presented in Figure 3B, whereas a calibration curve in the sub-femtomolar range is depicted in Figure $3 C$. The $L O D_{x}$ was calculated as described above and resulted in a value of 91.5 aM. This suggests that the established digital ELONA is perfectly suited for detecting longer ssDNA targets. An example of microscopy images of the microwell array for different concentrations is shown in Supplementary information (Figure S3).
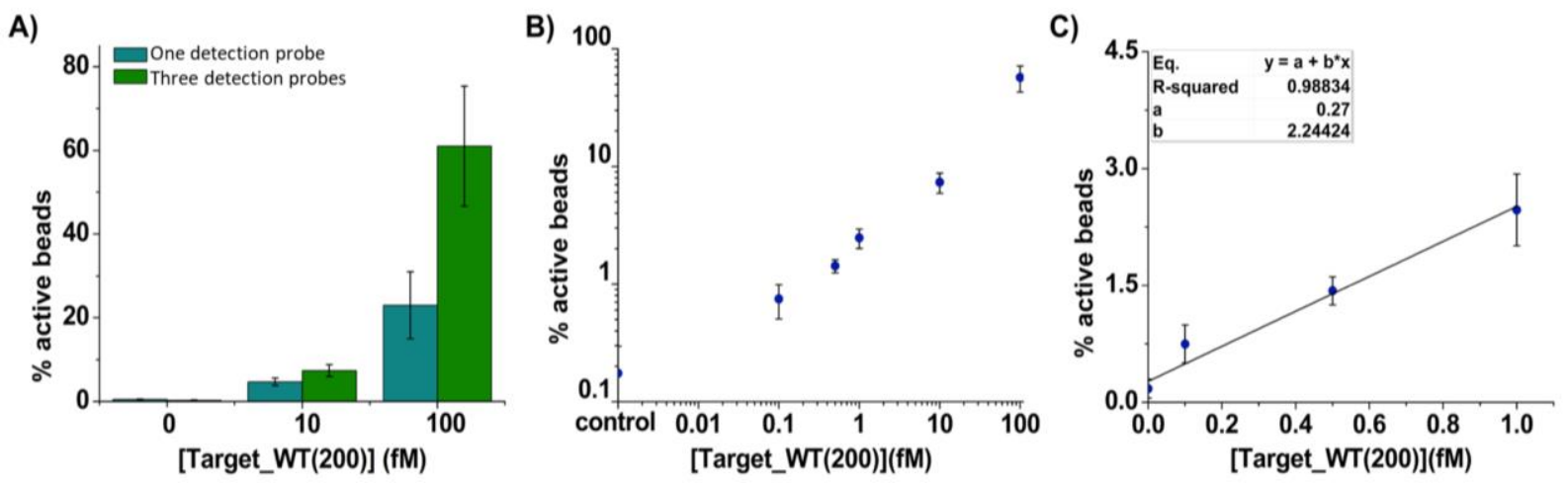

Figure 3: A) Percentage of active beads shown for different Target_WT (200) concentrations (0, 10 and $100 \mathrm{fM})$ in the presence of one (Detection probe 1_WT (200)) and three detection probes. B) Log-log plot of the percentage of active beads in 
function of femtomolar target concentrations. C) Calibration curve represents percentage of active beads in function of subfemtomolar target concentrations. The control signal ( $0.175 \%)$ corresponds to the non-specific binding of SBG enzyme to the magnetic beads at $0 \mathrm{fM}$ target concentration. Error bars represent one standard deviation based on three independent repetitions.

\subsection{Using HIH-microwell array for analog detection of higher target DNA concentrations}

As already shown in the previous sections, low ratios of target ssDNA molecules to beads (when detecting up to $100 \mathrm{fM}$ ) resulted in a significant number of beads not associated with a target molecule (and thus an SBG enzyme), which led to the number of microwells without fluorescent signal. Therefore, the ratio of active (i.e. fluorescent) beads with respect to the total number of beads seeded on the HIH-microwell array was always used to calculate the ratio of active beads. In those cases, the concentration of the DNA target was determined by counting the number of active beads, which was independent of the fluorescence intensity of the individual microwells (so called 'digital' detection approach).

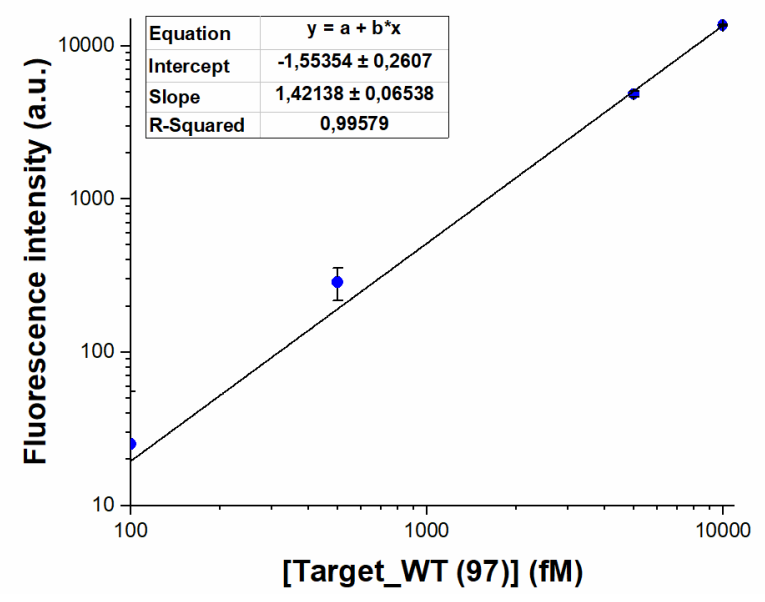

Figure 4: Analog detection of Target_WT (97) concentration between $100 \mathrm{fM}$ and $10 \mathrm{pM}$. Log-log plot of the average fluorescent intensity (a.u., arbitrary units) against the target DNA concentration. A linear fit is added $\left(R^{2}=0.99\right)$. Error bars represent standard deviations based on three repetitions. A log scale representation is chosen because of the wide range of the fluorescence intensities and concentrations. 
However, at higher concentrations of target ssDNA molecules, most beads typically capture more than one target, making accurate digital quantification impossible. Nevertheless, higher concentrations of target ssDNA molecules can potentially still be quantified using the same HIH-microwell array platform by measuring the average fluorescence intensity of an ensemble of microwells. This detection principle can then be referred to as 'analog' detection. To evaluate this, we measured the average fluorescence intensity on the HIH-microwell arrays for target ssDNA concentrations higher than $100 \mathrm{fM}$ (being 100, 500,5000 and $10000 \mathrm{fM}$ ) where typically $>80 \%$ of microwells containing a bead were fluorescent. For these measurements, a background image (taken immediately after FDG printing) was subtracted from a fluorescence image taken 5 min later. The average fluorescence intensity of the image was then plotted in function of the corresponding target ssDNA concentration and the obtained calibration curve is shown in Figure 4. These results indicate that the same HIH-microwell array technology can detect a large range of target ssDNA concentrations, spanning from aM to $\mathrm{pM}$ concentrations, which was recently proven when using the same technology platform for detecting protein biomarkers [33].

\subsection{Detection of SNPs using ligation-assisted digital ELONA}

To develop a technology platform that can enable not only sensitive detection of ssDNA molecules but also DNA molecules carrying SNPs (i.e. mutant strands), we established a novel concept, named ligation-assisted (LA) digital ELONA (Figure 5). Similar to the classical assay (Figure 1), target ssDNA molecules were also here captured first on magnetic beads, functionalized with complementary capture probes (Capture probe_LIG (97), Table 1), followed by hybridization of a biotinylated detection probe. However, in order to distinguish Target_MT (97) from the Target_WT (97) when they are both present in the mixture, capture and detection probes were ligated in a next step by a DNA Ligase. Due to the intrinsic high specificity of the ligase enzyme, ligation of capture and detection probe did not 
occur if a point mutation was present in the target DNA at the site of ligation [34, 35]. Therefore, the detection probes hybridized to the Target_MT (97) were dislodged by optimized heating and washing steps (Figure S4, Supplementary information), while the detection probes hybridized to the Target_WT (97) remained, thereby allowing binding of S $\beta$ G enzyme and detection of WT target only. Fluorescent readout was finally performed as described in previous paragraphs, enabling digital counting of Target_WT (97). It is important to note that, in order to achieve the ligation, capture probes were modified for this assay at their $5^{\prime}$ end with a phosphate group. This is fundamental for the ligase enzyme to create a phosphodiester bond between the phosphate group at the $5^{\prime}$ end of the capture probe and the hydroxyl group at the $3^{\prime}$ end of detection probe. As in the classical digital ELONA, an appropriate ratio between target molecules and magnetic beads allowed for detection of a singlemolecule per bead.

A)

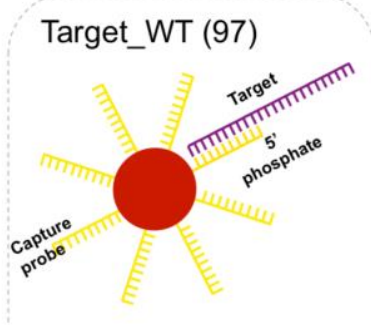

B)
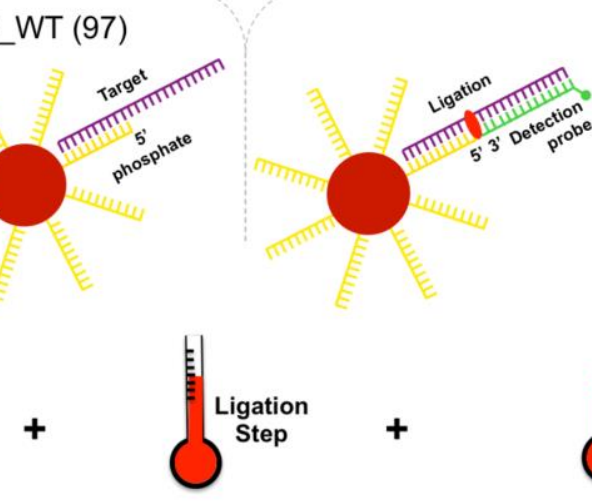

C)

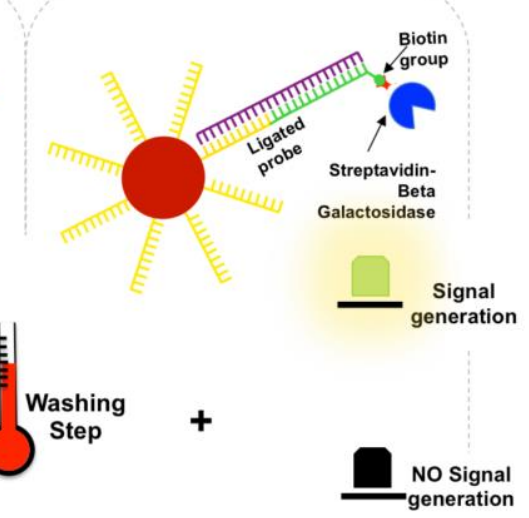

Target_MT (97)
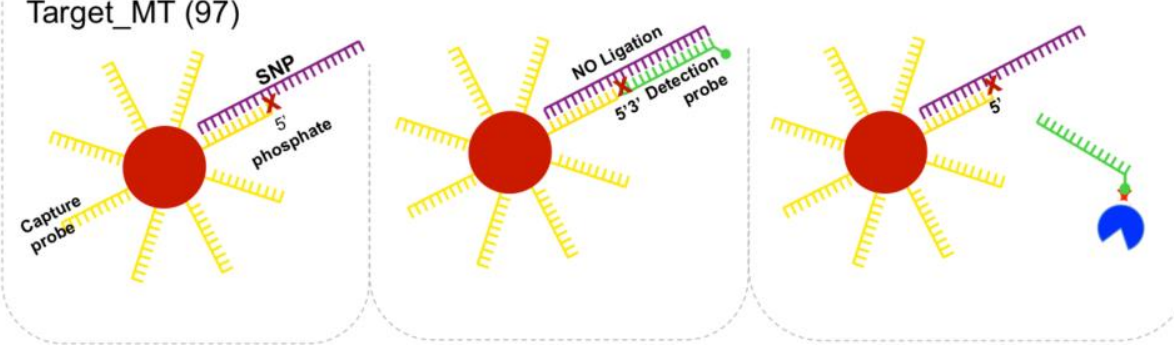
Figure 5: Schematic representation of LA digital ELONA for discriminating Target_WT (97) from Target_MT (97) when both are present in a mixture. A) Both MT and WT strands are captured on magnetic beads functionalized with capture probes (Capture probe_LIG (97)) in a single-molecule/bead configuration. B) Biotinylated detection probes are hybridized to the target strands next to the capture probes; ligation happens if the 3' nucleotide of the detection probe matches with the underling nucleotide of the strand (which is the case for Target_WT (97) but not for Target_MT (97)). C) Due to the presence of the SNP in Target_MT (97), heating and washing steps dislodge the not-ligated detection probes of the MT, allowing for fluorescent detection of only Target_WT (97).

Using the established ligation protocol (Figure S4, Supplementary information), samples were prepared for LA digital ELONA by mixing Target_WT (97) and Target_MT (97) in different ratios, being 2, 20 and $100 \mathrm{fM}$ concentration of Target_WT (97) in the final concentration of $200 \mathrm{fM}$. The percentage of active beads obtained for the three samples using LA digital ELONA is shown in Figure 6.

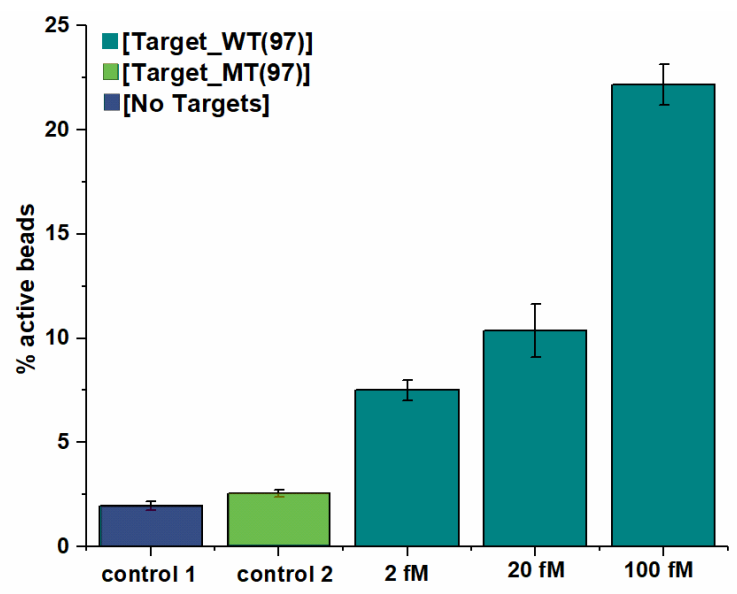

Figure 6: Percentage of active beads obtained for LA digital ELONA detection of Target_WT (97) when present at different concentrations in the mixture with Target_MT (97) and at the total DNA concentration of $200 \mathrm{fM}$. Error bars represent one standard deviation based on three independent measurements. 
These results demonstrate the capacity of the established assay to distinguish WT DNA strands from those carrying SNPs even when only $1 \%$ of WT strands was present in the DNA mixture and at a concentration as low as $2 \mathrm{fM}$. All the measurements were accompanied with two controls: control 1, containing no target DNA ( $0 \mathrm{fM}$ target) and control 2, containing $100 \mathrm{fM}$ of only Target_MT (97), the latter being important for controlling the efficiency of both ligation and washing steps. As shown in Figure 6 , both ligation at $50{ }^{\circ} \mathrm{C}$ and washing steps at $65^{\circ} \mathrm{C}$ seemed to be optimal for the assay since signals obtained for control 2 and control 1 were comparable, while being significantly different from signal obtained for the lowest concentration of the Target_WT (97).

Although this particular assay concept was intended for direct detection of WT DNA targets (by designing capture and detection probes complementary to the WT strand and knowing the exact location of the mutation), the same assay concept can be easily used for direct detection of DNA strands carrying SNPs, by designing capture and detection probes complementary to the MT strands. This would, however, require the knowledge on the exact position of SNPs within the MT target (in order to appropriately design the capture and detection probes that can match this), which is often known when aiming to detect specific DNA targets carrying SNPs, but no information on the type of mutation is needed.

\section{Conclusion}

In this paper, we developed two different types of bead-based digital bioassays using HIH-microwell array technology $[24,25]$ : digital ELONA for detecting ssDNA molecules and LA digital ELONA for discriminating WT DNA strands from those carrying SNPs. To establish digital ELONA, we first optimized several important bioassay parameters, such as the usage of three detection probes, to increase the sensitivity of the assay, as well as the usage of dextran sulfate, to improve the hybridization efficiency. With the optimized bioassay, we succeeded in detecting 97 nt-long ssDNA strands down to 68 aM, 
which was comparable to the previously reported sensitivity of $\mathrm{SiMoA}^{\mathrm{TM}}$ technology [18] In addition, we examined for the first time flexibility of the established digital ELONA concept towards detecting ssDNA molecules of different lengths. By designing longer capture and detection probes, to achieve better control over DNA target secondary structures, we reached LOD of 92 aM, proving that the presented concept is rather robust and can be used for detecting ssDNA targets of different lengths. However, since the design of detection probes is fundamental for a successful dELONA, a prior knowledge of possible secondary structures formation is needed for each specific target in order to optimize length of detection probes.

In addition, we demonstrated that the same HIH-microwell array platform can be used not only for implementing bead-based digital ELONA to detect sSDNA molecules present in femtomolar and sub-femtomolar concentrations, but as well for detecting targets present in much higher concentrations (up to picomolar) by using the so called 'analog' approach. This was in agreement with our previously published work showing the same features when detecting protein biomarkers [25]. Finally, on the same platform, we developed a new LA digital ELONA concept that enabled the discrimination of WT sequences from those carrying SNPs when both were present in a mixture. This bioassay was empowered by the unique intrinsic specificity of a ligation enzyme, which cannot form the phosphodiester bond if a point mutation is present in the target DNA at the site of ligation. We optimized the bioassay conditions towards ligation at $50{ }^{\circ} \mathrm{C}$ and washing steps at $65{ }^{\circ} \mathrm{C}$ and reached highly specific and sensitive detection of WT target DNA at concentrations as low as $2 \mathrm{fM}$ in the presence of 100 -fold higher concentrations of MT strands. Interestingly, the LA digital ELONA concept is very flexible and can be equally well used for direct detection of DNA strands carrying SNPs by simply designing capture and detection probes complementary to the MT strands.

In summary, the results presented in this manuscript indicate that the proposed method is highly sensitive, specific and very versatile towards different applications. Moreover, since the $\mathrm{HIH}-$ 
microwell array can be easily implemented on DMF chips [23], the proposed method perfectly fits in the technological context of future biosensor automation and miniaturization.

\section{Acknowledgements and founding sources}

We gratefully acknowledge financial support from KU Leuven (IOF/KP/12/Atheromix; OT 13/058; C32/15/005; C32/17/007), and Research Foundation-Flanders (FWO G086114N, G080016N, G0A6315, G084818N) and VLK (C8744). This project has received funding from the European Union's Horizon 2020 research and innovation programme under the Marie Skłodowska-Curie grant agreement No 675412 (H2020-MSCA-ITN-ND4ID) and No 764281 (H2020-MSCA-ITN-AiPBAND).

\section{References}

[1]. C.De Abreu Fonseca et al., Leptospira DNA detection for the diagnosis of human leptospirosis. Journal of Infection. 2006; $52 ; 1 ; 15-22$.

[2]. S.Nakamura et al., Metagenomic Diagnosis of bacterial infection. Emerg Infect Dis. 2008 ; 14 (11); 1784-1786.

[3]. S. Warwick et al., Use of Quantitative 16S Ribosomal DNA Detection for Diagnosis of Central Vascular CatheterAssociated Bacterial Infection. J Clin Microbiol. 2004; 42 (4); 1402-1408.

[4]. Xiao Han et al., Circulating Tumor DNA as Biomarkers for Cancer Detection. Genomics, Proteomics \& Bioinformatics. $2017 ; 15 ; 2 ; 59-72$.

[5]. A.A. Ensafi et al., Sensitive DNA impedance biosensor for detection of cancer, chronic lymphocytic leukemia, based on gold nanoparticles/gold modified electrode. Electrochimica Acta. 2011; 56; 24; ,8176-8183.

[6]. EM. Shaikho et al., A phased SNP-based classification of sickle cell anemia HBB haplotypes. BMC Genomics. 2017; 18:608. DOI:10.1186/s12864-017-4013-y.

[7]. FM. Gisler et al., Identification of SNPs in the cystic fibrosis interactome influencing pulmonary progression in cystic fibrosis. Eur J Hum Genet. 2013; 21(4); 397-403. 
[8]. L.A. Tortajada-Genaro et al., Multiplex DNA detection of Food Allergens on a digital Versatile Disk. Journal of Agricultural and Food Chemistry. 2012; 60 (1); 36-43.

[9]. A. Rasooly and K.E. Herold. Food Microbial Pathogen Detection and Analysis Using DNA Microarray Technologies. Foodborne Pathog Dis. 2008 ; 5(4); 531-550.

[10]. A. A. Hoffmann \& Y. Willi. Detecting genetic responses to environmental change. Nature Reviews Genetics. 2008; $9 ; 421-432$.

[11].P.Francis and T. E.Willerslev. Environmental DNA - An emerging tool in conservation for monitoring past and present biodiversity. Biological Conservation. 2015; 183; 4-18.

[12]. RJ. Pengelly et al. A SNP profiling panel for sample tracking in whole-exome sequencing studies. Genome Medicine. 2013; 5(9):89; DOI:10.1186/gm492.

[13]. D. Sims et al. Sequencing depth and coverage: key considerations in genomic analyses. Nature Reviews Genetics. $2014 ; 15 ; 121-132$.

[14]. J. M.S. Bartlett and D. Starling, PCR protocols, ed. John M. Walker Humana Press, edition 2. 2003; Chapter 25; $135-149$.

[15]. C. Larsson et al., In situ genotyping individual DNA molecules by target-primed rolling circle amplification of padlock probes. Nature Methods. 2004; 1; 227 - 232.

[16].M. Kühnemund et al., Sensitive and inexpensive digital DNA analysis by microfluidic enrichment of rolling single amplified single-molecule. Nucleic Acids Res. 2017; 45(8): e59; DOI:10.1093/nar/gkw1324.

[17]. CM. Clausson et al., Compaction of rolling single amplification products increases signal integrity and signal-to-noise ratio. Sci Rep. 2015; 5, DOI: 10.1038/srep12317.

[18]. IG. Wilson. Inhibition and facilitation of nucleic acid amplification. Applied and Environmental Microbiology. 1997; 63; 3741-3751.

[19].J . Maurer, Rapid detection and limitation of Molecular Techniques. Annual Review of Food Science and Technology. $2011 ; 2 ; 259-279$.

[20]. A. Borst et al., False-positive Results and Contamination in Nucleic Acid Amplification Assays: Suggestions for a Prevent and Destroy Strategy. European Journal of Clinical Microbiology and Infectious Diseases. 2004; 23; $289-299$.

[21]. L. Song et al., Direct detection of Bacterial genomic DNA at Sub-femtomolar Concentrations using single molecule arrays. Analytical Chemistry. 2013; 85; 1932-1939.

[22]. D. J. Reen, , Basic Protein and Peptide Protocols book, ed. John M. Walker, Humana Press, edition1. 1994; Chapter 47; 461-466. 
[23]. J. Crowther, Molecular Biomethods Handbook, ed. John M. Walker and Ralph Rapley, Humana Press, edition 2. 2008; Chapter 37;657-682.

[24]. D. W. Drolet, An enzyme-linked oligonucleotide assay. Nature biotechnology. 1996; 4; 1021-1025.

[25]. D. M. Rissin and D. R. Walt, Digital Concentration Readout of Single Enzyme Molecules Using Femtoliter Arrays and Poisson Statistics. Nano Lett. 2006; 6; 520-523.

[26]. Yi Zhang and H.Noji, Digital Bioassays: Theory, Applications and Perspectives. Anal.Chem. 2017; 89; 92-101.

[27]. D. M. Rissin DM. and D.R. Walt, Digital readout of target binding with attomol detection limits via enzyme amplification in femtoliter arrays. J Am Chem Soc. 2006; 128; 6286-6287.

[28]. D.M. Rissin et al., Single-molecule immunoassorbent assay detects serum proteins at sub-femtomolar concentrations. Nature Biotechnology. 2010; 28; 595-599.

[29]. C. W. Kan et al., Isolation and detection of single molecules on paramagnetic beads using sequential fluid flows in microfabricated polymer array assemblies. Lab on Chip. 2012; 12;5; 977-985.

[30]. H. Zhang et al., Oil-sealed femtoliter fiber-optic arrays for single molecule analysis. Lab Chip. 2012; 12; $2229-2239$.

[31]. D. Witters et al., Digital Microfluidics enabled single-molecule detection by printing and sealing single magnetic beads in femtoliter droplet. Lab on a Chip. 2013; 13; 2047-2054.

[32]. K. Leirs et al., Bioassay Development for Ultrasensitive detection of Influenza A Nucleoprotein Using Digital ELISA. Anal. Chem. 2016; 88; 17; 8450-8458.

[33]. E. Pérez Ruiz et. al, Digital ELISA for the quantification of attomolar concentrations of Alzheimer's disease biomarker protein Tau in biological samples. Analytica Chimica Acta. 2018; 1015; 74-81.

[34]. F. Barany, Genetic disease detection and DNA amplification using cloned thermostable ligase. Proc. Natl. Acad. Sci U S A. $1991 ; 88(1) ; 189-193$.

[35]. P. Hardenbol et al., Multiplexed genotyping with sequence-tagged molecular inversion probes. Nature Biotechnology. $2003 ; 21 ; 673-678$. 